\title{
Pensamiento crítico en estudiantes de educación básica
}

\author{
Mendoza Cruzado Dante Robinson \\ mcruzadodr@ucvvirtual.edu.pe \\ https://orcid.org/0000-0002-2785-7165 \\ Universidad César Vallejo, Perú
}

\section{RESUMEN}

Este artículo tiene por finalidad evidenciar la revisión sistemática de diversas investigaciones referidas al tema de estudio, como consecuencia de una problemática evidente en el sistema educativo, que aún se encuentra arraigado con los métodos tradicionales de enseñanza-aprendizaje. Ha surgido, de esta manera, comprender y aprehender que el pensamiento crítico es de vital importancia que, mediante su aplicación, se despertará en los estudiantes diversos criterios como lo son la creatividad, la innovación, el compromiso con su propia educación y la mejora de su rendimiento académico. Para el desarrollo del presente trabajo se ha recurrido a fuentes avaladas por los buscadores más confiables como Google académico, Scopus, EBSCO Host y ProQuest. Además, se ha tenido en cuenta los criterios de inclusión, en referencia a la indagación de los artículos científicos, considerando únicamente aquellos cuya publicación no sea mayor a los 3 años. En lo referido a la clasificación de los artículos escogidos, éstos fueron determinados conforme a los controles y criterios para su selección, rigurosamente seguidos que garantizan la calidad de los mismos. Por lo mencionado, surgió como problema de la investigación: ¿Bajo qué perspectiva está orientado el pensamiento crítico en la educación básica? Por tanto, el presente artículo pretende crear una discusión sobre la visión que se le viene dando a la promoción y el inventivo del pensamiento crítico como parte del desarrollo de las habilidades de los educandos, especificando a las metodologías empleadas como variable principal del análisis realizado.

Palabras clave: pensamiento crítico; enseñanza - aprendizaje; educación; habilidades. 


\title{
Critical thinking in basic education students
}

\begin{abstract}
The purpose of this article is to demonstrate the systematic review of various investigations related to the subject of study, as a consequence of an evident problem in the educational system, which is still rooted in traditional teaching-learning methods. In this way, it has emerged to understand and apprehend that critical thinking is of vital importance that, through its application, will awaken in student's various criteria such as creativity, innovation, commitment to their own education and improvement of their academic performance. Mention that, for the development of this work, we have resorted to sources endorsed by the most reliable search engines such as academic Google, Scopus, EBSCOhost and ProQuest. In addition, the inclusion criteria have been taken into account, in reference to the investigation of scientific articles, considering only those whose publication is not older than 3 years. Regarding the classification of the chosen articles, they were determined according to the controls and criteria for their selection, rigorously followed to guarantee their quality. Therefore, it emerged as a research problem: Under what perspective is critical thinking oriented in basic education? Therefore, this article aims to create a discussion about the vision that has been given to the promotion and inventiveness of critical thinking as part of the development of the skills of students, specifying the methodologies used as the main variable of the analysis carried out.
\end{abstract}

Key words: critical thinking; teaching - learning; education; skills

Artículo recibido: 02 noviembre. 2021 Aceptado para publicación: 28 noviembre 2021 Correspondencia: amcruzadodr@ucvvirtual.edu.pe Conflictos de Interés: Ninguna que declarar 


\section{INTRODUCCIÓN}

El presente artículo plantea como principal interrogante ¿Bajo qué perspectiva está orientado el pensamiento crítico en la educación básica?, dado que en el contexto actual, los promotores de la educación han señalado la importancia de incluir el desarrollo del pensamiento crítico desde los primeros niveles de educación; es decir, desde la infancia, dado que, el niño o la niña están propensos a la adquisición no solo de conocimientos, sino de ser capaces de resolver sus problemas a través de un pensamiento crítico, aún con sus limitaciones, pero que, al ser motivados y orientados al desarrollo de éste, se les facilitará el manejo de sus habilidades sociales y emocionales en el transcurso de su desarrollo.

Este método de investigación, tiene como punto de referencia el análisis crítico que se realiza en base a un tema en específico. En lo referido a la clasificación de los artículos escogidos, éstos fueron determinados conforme a los controles y criterios para su selección, rigurosamente seguidos que garantizan la calidad de los mismos. Por tanto, el presente artículo pretende crear una discusión sobre la visión que se le viene dando a la promoción y el inventivo del pensamiento crítico como parte del desarrollo de las habilidades del educando, especificando a las metodologías empleadas como variable principal del análisis realizado.

En referencia a las investigaciones que fueron seleccionadas para desarrollar el artículo, tenemos al estudio de Castillo (2020), el autor resalta la importancia de relevar el pensamiento crítico, de forma adecuada y eficiente, para conducir al logro de los objetivos fundamentales de las instituciones educativas, de ahí que se deba integrar debidamente el ejercicio crítico de análisis, dilucidación, deliberación y síntesis de los conocimientos adquiridos, solo de este modo, los fines educativos trazados, actuarán de manera retroactiva señalando significados relevantes para el aprendizaje de los /as estudiantes.

Asimismo, Núñez, Gallardo, Aliaga y Díaz (2020), pudieron evidenciar que la acción pedagógica influye de manera significativa en la formación de personas autónomas que pasan de la simple recepción de información al procesamiento de ella. Esta acción pedagógica induce a los estudiantes a explorar e inquirir en el campo de lo poco conocido con interés, buscando permanentemente razones, respuestas o conocimientos que esclarezcan las dudas e inquietudes en el campo del saber que poseen. De los resultados ofrecidos por este estudio, se puede apreciar la necesidad de establecer el replanteamiento 
de las políticas educativas, reorientando los procesos de la formación continua de los docentes y el diseño curricular escolar. Además de priorizar las nuevas prácticas pedagógicas, con soporte tecnológico a través del uso de las TIC, utilizando técnicas como el coaching, profundizando el nivel de conocimiento cognitivo y, para el segundo, que fortalezca las habilidades duras, muy dejadas a menos por la priorización de las habilidades blandas.

\section{MÉTODOS}

La revisión de la literatura implica detectar, consultar y obtener la bibliografía (referencias) y otros materiales que sean útiles para los propósitos del estudio, de donde se tiene que extraer y recopilar la información relevante y necesaria para enmarcar nuestro problema de investigación. Esta revisión debe ser selectiva, puesto que cada año se publican en el mundo miles de artículos en revistas académicas y periódicos, libros y otras clases de materiales sobre las diferentes áreas del conocimiento. Si al revisar la literatura nos encontramos con que en el área de interés hay 5000 referencias, es evidente que se requiere seleccionar sólo las más importantes y recientes, y que además estén directamente vinculadas con nuestro planteamiento del problema de investigación. En ocasiones, revisamos referencias de estudios tanto cuantitativos como cualitativos, sin importar nuestro enfoque, porque se relacionan de manera estrecha con nuestros objetivos y preguntas.

A continuación, se comentan los pasos que se siguen para revisar la literatura. (Sampiere, 2014). Para el desarrollo de este artículo de revisión, se planteó como pregunta de investigación, la siguiente: ¿Bajo qué perspectiva está orientado el pensamiento crítico en la educación básica?

En referencia a la recolección de información y el proceso de búsqueda de los artículos científicos, se emplearon los buscadores como Scopus, EBSCO Host, Google académico y Pro Quest; como medios que facilitaron la obtención de datos relevantes para la investigación. Además, la búsqueda de artículos relevantes tuvo un periodo de tres meses, de octubre, 2020 a diciembre, 2020. Se consideraron como criterios para la búsqueda de dichos artículos, tales como palabras claves (Pensamiento crítico), y operadores booleanos (and), limitación (Texto completo), año de publicación (2017-2020), tipo de estudios (artículos de educación), idioma (español), entre otros que facilitaron la obtención de información precisa. 
- En Scopus; para iniciar la búsqueda se colocó como palabra clave *pensamiento crítico en educación*, y obteniendo como resultado 35 artículos, luego de aplicar el filtro años de investigación del 2017-2020, solo en idioma español y de educación quedando 13 artículos de los cuales 10 se ajustaron a los requerimientos de la investigación.

- En Pro Quest; para iniciar la búsqueda se colocó como palabra clave pensamiento crítico en la educación, delimitándose al rango de tiempo de 2016-2020, obteniéndose un total de 60 resultados, de los cuales fueron seleccionado 10 artículos científicos, las demás búsquedas descartadas debido a que se trataban de tesis en su mayoría y artículos incompletos o enfocados en otros aspectos del pensamiento crítico.

- En Google académico, para iniciar la búsqueda se colocó como palabra clave pensamiento crítico AND educación, y se obtuvo como resultado 58.900 resultados, se consideraron como criterios el período de publicación de 2017 al 2020, el idioma español, como siguientes criterios; para luego seleccionar solo diez (10) artículos que se fijaban a los criterios de investigación.

- En EBSCO host; para iniciar la búsqueda se colocó como palabra clave *pensamiento crítico y educación básica* obteniéndose 32.334 resultados, se consideró el período 2017-2020, obteniéndose 10.652, como otro criterio se consideró que éstos sean textos completos en pdf y revistas científicas, obteniendo 43 artículos, seleccionándose finalmente 10 artículos de investigación.

\section{Tabla 1}

Estrategias de búsqueda

\begin{tabular}{clc} 
Ítem & Buscador Académico & Criterio de Inclusión \\
$\mathbf{1}$ & Scopus & *pensamiento crítico en educación* \\
\hline $\mathbf{2}$ & Pro Quest & pensamiento crítico en la educación \\
\hline $\mathbf{3}$ & Google Académico & pensamiento crítico AND educación \\
\hline $\mathbf{4}$ & EBSCO host & *pensamiento crítico y educación básica* \\
\hline
\end{tabular}

Nota. Elaboración propia 


\section{RESULTADOS}

En esta investigación fueron escogidos de manera equitativa 10 artículos por cada buscador sobre el tema a investigar, con la finalidad de explorar diversos buscadores e investigaciones que se han realizado sobre el tema, como se muestra a continuación en el siguiente gráfico:

Figura 1. Porcentaje de revisión en buscadores.

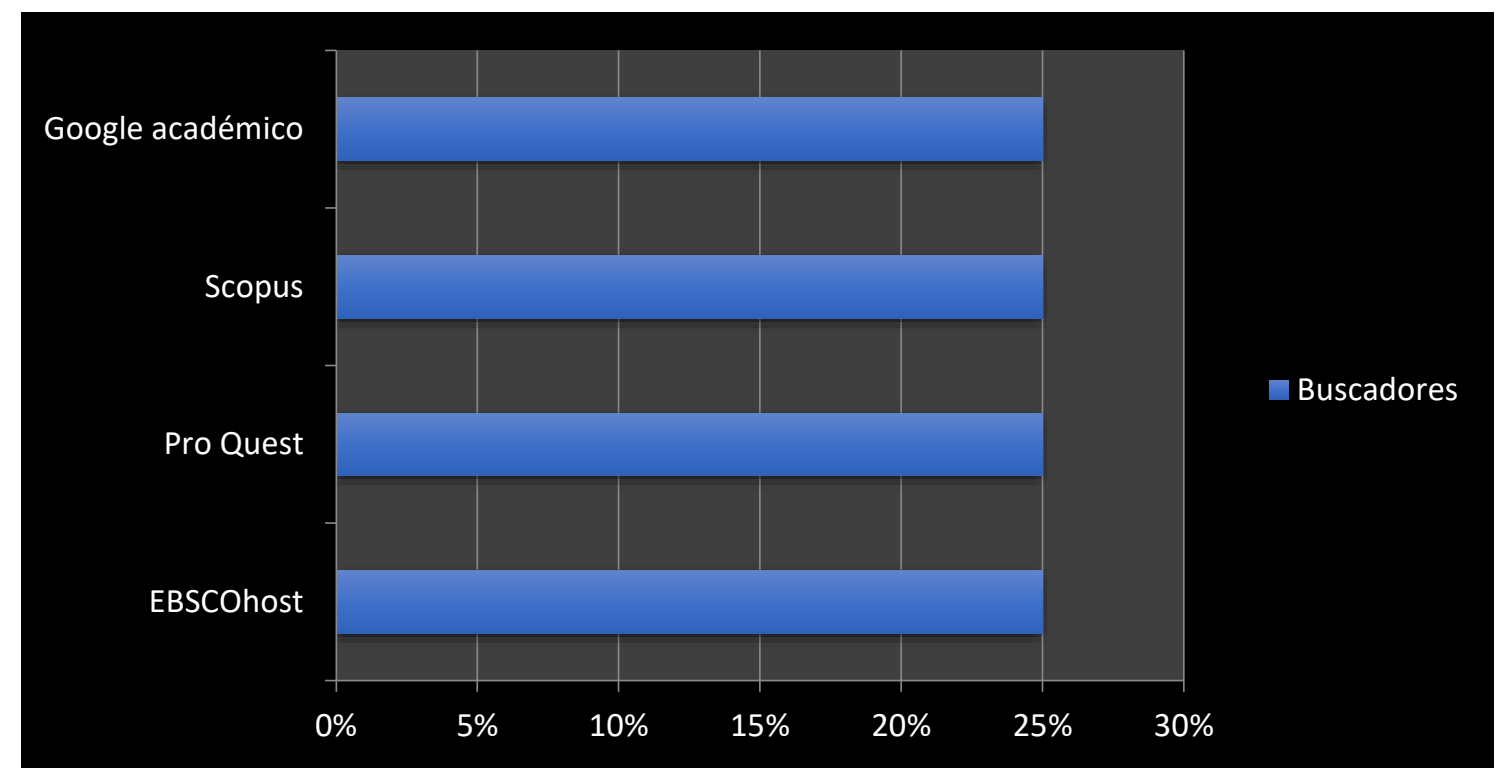

La problemática entorno al desarrollo del pensamiento crítico en la educación básica ha sido desarrollada por autores de países no solo de Latinoamérica sino de Europa, como es el caso de España, Rodríguez (2018), cuya investigación señala que en la base natural del pensamiento crítico se puede encontrar tres elementos que constituyen su ontología: lenguaje, pensamiento y acción. Elementos que permiten el desarrollo de habilidades de nivel superior para razonar, tomar decisiones y actuar de manera racional y razonable. Se destaca la importancia del lenguaje como elemento central y eje relacional entre el pensamiento y la acción es el que hace posible alcanzar grados superiores de racionalidad, intencionalidad y conciencia y, con ello, permite alcanzar ciertas estructuras del pensamiento para pensar y actuar críticamente.

Así mismo se evidencian, investigaciones de diversos países, como Argentina, Brasil, Ecuador, Chile, México, Colombia, España y Perú. 
Figura 2. Artículos por país de publicación

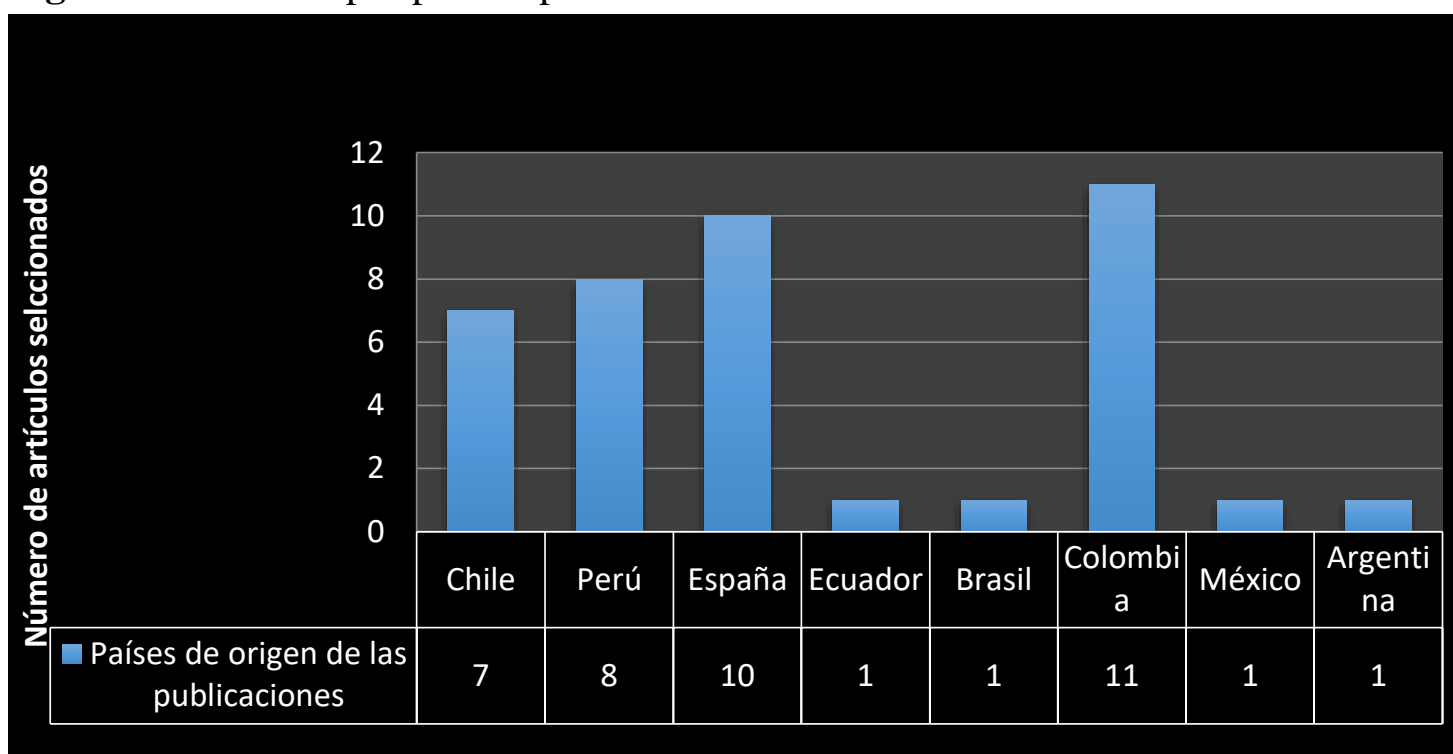

Nota. Elaboración en base a búsquedas realizadas en Scopus, Google académico, EBSCO Host y ProQuest.

En su mayoría, se pudo evidenciar que los artículos hallados fueron de tipo cualitativos, y en una menor proporción, descriptivas, cualitativas y mixtas. Así se tiene que un $75 \%$ de los artículos de investigación tuvieron un enfoque cualitativo, por lo que las investigaciones halladas constituyen un análisis documental con diseño cuasi experimentales y análisis documental. Además, un $10 \%$ son investigaciones con enfoque cuantitativos, cuya finalidad fue medir por medio de instrumentos el proceso de aprendizaje de los estudiantes en referencia a su desarrollo del Pensamiento Crítico; un $10 \%$ fueron investigaciones mixtas y un 5\% investigaciones descriptivas. Dentro de las investigaciones los autores presentan en su mayoría propuestas creadas por ellos mismos con el fin de impulsar el desarrollo del pensamiento crítico de los estudiantes en el proceso de enseñanza-aprendizaje.

Figura 3. Artículos por tipo de investigación.

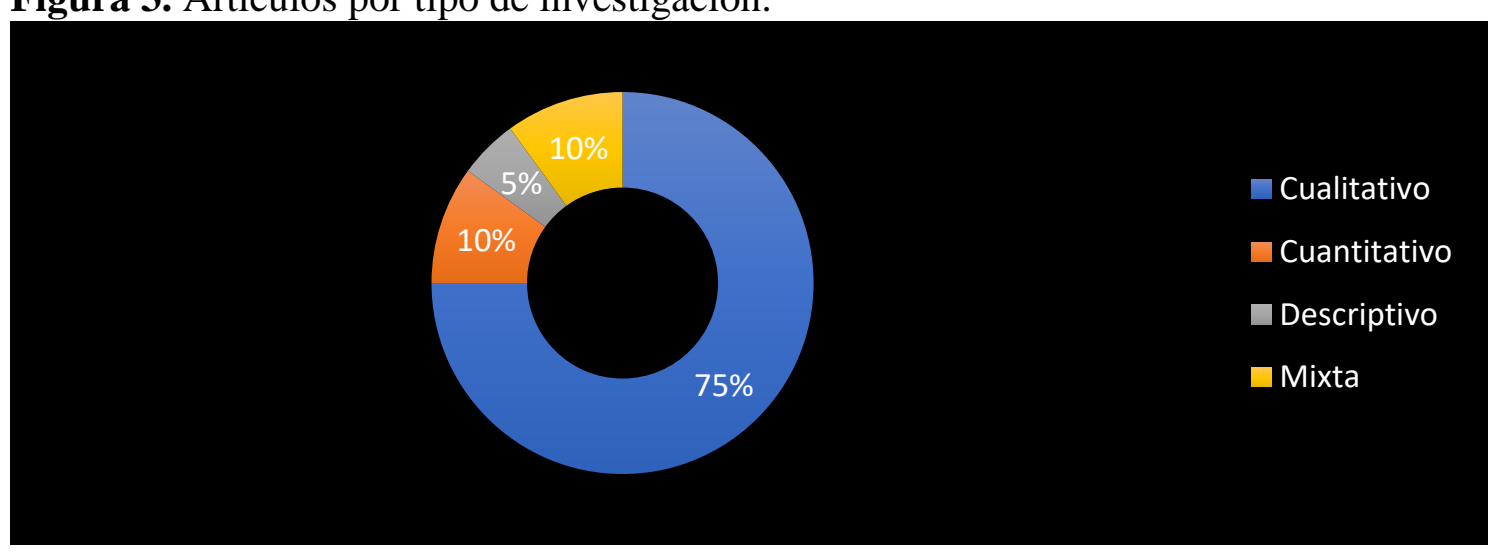

Nota. Elaboración en base a búsquedas realizadas en Scopus, Google académico, EBSCO Host y ProQuest. 
Dante Robinson...

En la tabla siguiente se muestra los diversos autores consultados con su aporte sobre el tema tratado, contribuyendo de manera significativa al amplio discurso sobre el desarrollo del pensamiento crítico en la educación básica.

Tabla 2 Aportes de los autores respecto al tema de la investigación

\begin{tabular}{|c|c|c|c|}
\hline & Autor (es) & Título & Motivos de inclusión \\
\hline 1 & Castillo, R. & $\begin{array}{l}\text { El Pensamiento } \\
\text { Crítico como } \\
\text { competencia } \\
\text { básica. } \\
\text { Una propuesta de } \\
\text { nuevos } \\
\text { estándares } \\
\text { pedagógicos. }\end{array}$ & $\begin{array}{l}\text { Este estudio resalta la importancia de relevar el pensamiento } \\
\text { crítico, de forma adecuada y eficiente, para conducir al logro } \\
\text { de los objetivos fundamentales de las instituciones } \\
\text { educativas de Chile, de ahí que se deba integrar debidamente } \\
\text { el ejercicio crítico de análisis, dilucidación, deliberación y } \\
\text { síntesis de los conocimientos adquiridos, solo de este modo, } \\
\text { los fines educativos trazados, actuarán de manera retroactiva } \\
\text { señalando significados relevantes para el aprendizaje de los } \\
\text { /as estudiantes. }\end{array}$ \\
\hline 2 & $\begin{array}{c}\text { Deroncele, } \\
\text { A., } \\
\text { Nagamine, } \\
\text { M. y } \\
\text { Medina, D. }\end{array}$ & $\begin{array}{l}\text { Desarrollo del } \\
\text { pensamiento } \\
\text { crítico }\end{array}$ & $\begin{array}{l}\text { Los autores determinaron que en el Perú las propuestas para } \\
\text { la aplicación de estrategias o programas para el DPC están } \\
\text { relacionados a investigaciones de carácter individual } \\
\text { producto de tesis de posgrado principalmente. No se han } \\
\text { hallado propuestas a nivel macro, producto de } \\
\text { investigaciones teóricas y aplicadas, que atiendan a los } \\
\text { diversos niveles y contextos educativos existentes en el país, } \\
\text { observándose una oportunidad de trabajo investigativo, con } \\
\text { el propósito de desarrollar este tipo de pensamiento tan } \\
\text { relevante en el mundo actual, en los estudiantes peruanos. }\end{array}$ \\
\hline 3 & $\begin{array}{c}\text { Moreno, W. } \\
\text { y } \\
\text { Velázquez, } \\
\text { M. }\end{array}$ & $\begin{array}{c}\text { Estrategia } \\
\text { Didáctica para } \\
\text { Desarrollar el } \\
\text { Pensamiento } \\
\text { Crítico }\end{array}$ & $\begin{array}{l}\text { Los autores determinaron que, para enfrentar los desafíos y } \\
\text { los retos de la educación en la actualidad, se necesita que la } \\
\text { escuela potencie en los estudiantes el desarrollo del } \\
\text { pensamiento crítico de manera que puedan estar en } \\
\text { condiciones para enfrentarse a los diversos problemas de su } \\
\text { contexto de actuación y puedan contribuir a la } \\
\text { transformación de la realidad. El análisis cualitativo y } \\
\text { cuantitativo del diagnóstico de campo evidenció que los } \\
\text { estudiantes al realizar las actividades de aprendizaje no } \\
\text { analizan la información, no saben proponer alternativas de } \\
\text { solución y reflejan un pensamiento productivo alejado del } \\
\text { ejercicio de la crítica. }\end{array}$ \\
\hline 4 & $\begin{array}{c}\text { Botero, A.; } \\
\text { Alarcón, D. } \\
\text { Palomino, } \\
\text { D. y } \\
\text { Jiménez, A. }\end{array}$ & $\begin{array}{c}\text { Pensamiento } \\
\text { crítico, } \\
\text { metacognición } \\
\text { y aspectos } \\
\text { motivacionales: } \\
\text { una } \\
\text { educación de } \\
\text { calidad }\end{array}$ & $\begin{array}{l}\text { El estudio determina que, en el contexto educativo actual, } \\
\text { independientemente del nivel educativo, desarrollar el } \\
\text { pensamiento crítico en los sujetos conlleva a generar } \\
\text { acciones que favorecen el rigor intelectual y el aprendizaje } \\
\text { autónomo. El pensamiento crítico posibilita transformar la } \\
\text { información proveniente de la realidad social, analizar } \\
\text { afirmaciones para evaluar su precisión, pertinencia, validez } \\
\text { y a su vez, elaborar juicios críticos acorde al contexto, en el } \\
\text { cual se encuentran inmersos. La misión del contexto } \\
\text { educativo, debe ser entonces: atender el desarrollo de } \\
\text { destrezas de orden superior como las del pensamiento crítico } \\
\text { que propendan por el aprender a aprender; procurando así, } \\
\text { que el estudiante llegue a adquirir una autonomía intelectual. }\end{array}$ \\
\hline
\end{tabular}




\begin{tabular}{|c|c|c|c|}
\hline 5 & $\begin{array}{l}\text { Ortega, V.; } \\
\text { Gil, C.; } \\
\text { Valles, C. y } \\
\text { López, M. }\end{array}$ & $\begin{array}{l}\text { Diseño y } \\
\text { validación de } \\
\text { instrumentos de } \\
\text { evaluación de } \\
\text { pensamiento } \\
\text { crítico en } \\
\text { educación } \\
\text { primaria }\end{array}$ & $\begin{array}{l}\text { Para los autores, el Pensamiento Crítico contempla } \\
\text { cuestiones fundamentales del pensamiento científico desde } \\
\text { una perspectiva general, pensada para ser aplicada en la vida } \\
\text { cotidiana. Este planteamiento coincide con la necesidad de } \\
\text { desarrollo de estas capacidades en la población, } \\
\text { especialmente en los jóvenes, y de ahí la necesidad de su } \\
\text { inclusión y promoción en los contextos educativos. }\end{array}$ \\
\hline 6 & $\begin{array}{c}\text { Gómez, D. y } \\
\text { De la } \\
\text { Herrán, A. }\end{array}$ & $\begin{array}{l}\text { Desarrollo del } \\
\text { pensamiento } \\
\text { crítico en } \\
\text { estudiantes de } \\
\text { educación } \\
\text { secundaria: } \\
\text { diseño, } \\
\text { aplicación y } \\
\text { evaluación de un } \\
\text { programa } \\
\text { educativo }\end{array}$ & $\begin{array}{l}\text { La realización de este programa ha supuesto una oportunidad } \\
\text { para cambiar la metodología docente, dando la posibilidad a } \\
\text { los estudiantes de desarrollar su pensamiento crítico, de } \\
\text { reflexionar científicamente en clase, de trabajar con } \\
\text { situaciones reales y de contribuir a la construcción de } \\
\text { determinadas herramientas intelectuales que les permitan } \\
\text { estar en disposición de tomar las decisiones adecuadas en } \\
\text { una sociedad tan dinámica y alienante como la actual. }\end{array}$ \\
\hline 7 & $\begin{array}{c}\text { Del Barrio, } \\
\text { L. }\end{array}$ & $\begin{array}{l}\text { El Pensamiento } \\
\text { Crítico Como } \\
\text { Estrategia } \\
\text { Didáctica } \\
\text { Musical En La } \\
\text { Educación } \\
\text { Emancipatoria } \\
\text { Del Alumno En } \\
\text { Educación } \\
\text { Primaria }\end{array}$ & $\begin{array}{l}\text { El autor determinó que la promoción del pensamiento crítico } \\
\text { en el proceso educativo musical en Educación Primaria } \\
\text { integra la habilidad de pensar como una actividad natural de } \\
\text { autoaprendizaje que estimula la comunicación y la reflexión } \\
\text { como actitudes de acceso y análisis del conocimiento desde } \\
\text { las que se promueve la educación de la autonomía y la } \\
\text { madurez del estudiante. El docente como director del } \\
\text { proceso educativo debe clarificar los objetivos del trabajo, } \\
\text { promover el pensamiento y el diálogo en el aula, fomentar la } \\
\text { búsqueda de la verdad, la escucha activa y la flexibilidad, } \\
\text { facilitar herramientas metodológicas y de evaluación del } \\
\text { aprendizaje que permitan analizar su aplicabilidad y } \\
\text { reconocer y potenciar los valores que ayudan a la educación } \\
\text { emancipatoria del estudiante. }\end{array}$ \\
\hline 8 & $\begin{array}{l}\text { Tamayo, O., } \\
\text { Loaiza Y. y } \\
\text { Ruiz F. }\end{array}$ & $\begin{array}{c}\text { Hacia la } \\
\text { construcción de } \\
\text { un modelo de } \\
\text { pensamiento } \\
\text { crítico dominio } \\
\text { específico }\end{array}$ & $\begin{array}{l}\text { El estudio identifica la importancia del requerimiento de la } \\
\text { dimensión emocional para la constitución del pensamiento } \\
\text { crítico, de ahí que, la formación del pensamiento crítico } \\
\text { dominio-específico es uno de los objetos de estudio centrales } \\
\text { de la didáctica de las ciencias. La perspectiva teórica } \\
\text { asumida por los grupos de investigación Cognición y } \\
\text { Educación y Maestros y Contextos, profundiza en el estudio } \\
\text { de cuatro dimensiones: solución de problemas, } \\
\text { argumentación, emociones y metacognición, las cuales son } \\
\text { centrales en la formación del pensamiento crítico de los } \\
\text { estudiantes. }\end{array}$ \\
\hline 9 & $\begin{array}{c}\text { Correa, J.; } \\
\text { Ossa, C. y } \\
\text { Sanhueza, P. }\end{array}$ & $\begin{array}{l}\text { Sesgo en } \\
\text { razonamiento, } \\
\text { metacognición } \\
\text { y motivación al } \\
\text { pensamiento } \\
\text { crítico en } \\
\text { estudiantes de } \\
\text { primer año medio } \\
\text { de un } \\
\text { establecimiento } \\
\text { de Chillán }\end{array}$ & $\begin{array}{l}\text { Los autores señalan que es necesario entregar a los } \\
\text { estudiantes las herramientas adecuadas para estimular un } \\
\text { pensamiento crítico, creativo y metacognitivo, } \\
\text { características de un pensamiento productivo, puesto que } \\
\text { estas son habilidades fundamentales del siglo XXI. Sobre } \\
\text { esa base es posible intencionar un aprendizaje profundo de } \\
\text { los contenidos escolares, donde el estudiante sea capaz de } \\
\text { realizar múltiples operaciones con el conocimiento } \\
\text { adquirido y establecer relaciones disciplinares e } \\
\text { interdisciplinares de mayor complejidad. }\end{array}$ \\
\hline
\end{tabular}




\begin{tabular}{|c|c|c|c|}
\hline 10 & Madrid, H & $\begin{array}{c}\text { Desarrollo del } \\
\text { pensamiento } \\
\text { crítico desde el } \\
\text { área de } \\
\text { Ciencias Sociales } \\
\text { en la } \\
\text { educación básica } \\
\text { secundaria }\end{array}$ & $\begin{array}{l}\text { Se determinó en el estudio que, aunque el contexto demande } \\
\text { una educación distinta, creativa, que ubique al estudiante en } \\
\text { el centro del proceso educativo, puesto que es necesario } \\
\text { contar con un sujeto que participe activamente en las } \\
\text { decisiones y las transformaciones que el país necesita, de allí } \\
\text { que las instituciones educativas propongan unos modelos } \\
\text { educativos que en esencia pretenden formar ciudadanos y } \\
\text { ciudadanas con una mentalidad ajustada a las condiciones } \\
\text { actuales, en el sentido de que cuenten con las capacidades de } \\
\text { construir procesos sociales como seres educados y } \\
\text { civilizados. }\end{array}$ \\
\hline
\end{tabular}

Nota. Elaboración en base a búsquedas realizadas en Scopus, Google académico, EBSCO Host y

ProQuest.

Como se puede evidenciar, se ha recopilado información valiosa sobre los diversos enfoques que se le da al desarrollo del pensamiento crítico. En el cuadro anterior se plasmaron 10 aportes de los 40 artículos recopilados, dedicados y preocupados por el desarrollo, no solo cognitivo, sino crítico, emocional, creativo de los estudiantes, es decir, resaltan la importancia de la formación de futuros ciudadanos capaces de comprender y enfrentar los diversos retos de un mundo globalizado, y que les permita resolver situaciones problemáticas, analizando el contexto en el que se desarrollan y que su actuar refleje que han adquirido las habilidades necesarias para la vida.

Es deber de los docentes, a pesar de las carencias y dificultades del sistema, contribuir eficientemente con este proceso que desencadenará una serie de resultados positivos, no solo para los estudiantes sino para la sociedad. Tal como lo indica Godoy (2017), que las condiciones materiales de los centros educativos donde se desempeñan los profesores no siempre ofrecen las mejores posibilidades para la formación de buenos hábitos de pensamiento en los estudiantes. Además, es evidente la existencia de un problema de fondo de tipo estructural que no facilita la tarea de formar el pensamiento crítico. Los espacios físicos, la distribución del mobiliario, la gestión curricular del centro, las cargas horarias, las características de los estudiantes y sus contextos sociales y de vida, las responsabilidades que pesan sobre los docentes, entre otras, no favorecen los intentos por formar el pensamiento crítico en la escuela. No obstante, entendemos, que, en última instancia, es el profesorado quien deberá tomar las decisiones respecto a cómo gestionará el currículum, qué y cómo enseñará en el aula, dado que los programas de estudio plantean las orientaciones didácticas sólo a manera de sugerencia, entregando al docente la decisión final de su implementación. Es él o ella, en definitiva, quien decidirá qué sello imprimirá a su práctica. 


\section{DISCUSIÓN}

El estudio realizado sobre revisión sistemática de artículos analizando la producción científica sobre el pensamiento crítico en la educación básica, precisaron muchas estrategias metodológicas y para cada una de ellas sus correspondientes habilidades significando que las estrategias no se aplican directamente sino a través de habilidades. Esto conduce a trabajar y agotar el conocimiento de las habilidades; ello significa que se tienen que seguir pasos determinados, corresponde a un proceso, donde el docente aplique la evaluación formativa e inmediatamente aplicar la retroalimentación según sea necesaria. Los autores consultados concuerdan con trabajar el desarrollo del pensamiento crítico aplicando estrategias específicas, con diseños de investigación que respondan a la población de estudio.

Como precisa Del Barrio (2017), el pensamiento crítico tiene la capacidad de favorecer la autonomía intelectual del estudiante de educación primaria. La educación del pensamiento crítico implica un trabajo de la flexibilidad cognitiva, para admitir los cambios que puedan darse, mediante el entrenamiento de operaciones mentales como proponer, eliminar, combinar, sustituir, modificar, adaptar o reorganizar la información. Pensar críticamente ha conllevado salir de la propia subjetividad para escuchar los argumentos de los demás, analizar y evaluar de manera objetiva, con el fin de cambiar y mejorar el aprendizaje, y así transformar las condiciones de su entorno de acción.

Por su parte, Collazo (2017), señala que, la promoción del pensamiento crítico en el proceso educativo en Educación Primaria integra la habilidad de pensar como una actividad natural de autoaprendizaje que estimula la comunicación y la reflexión como actitudes de acceso y análisis del conocimiento desde las que se promueve la educación de la autonomía y la madurez del estudiante. El docente como director del proceso educativo debe clarificar los objetivos del trabajo, promover el pensamiento y el diálogo en el aula, fomentar la búsqueda de la verdad, la escucha activa y la flexibilidad, facilitar herramientas metodológicas y de evaluación del aprendizaje que permitan analizar su aplicabilidad y reconocer y potenciar los valores que ayudan a la educación emancipatoria del estudiante.

Según la revisión sistemática de este trabajo ha sido demostrado que a pesar de que el pensamiento crítico es un requisito esencial para la transformación del sistema educativo y avanzar hacia la sostenibilidad, no es por sí mismo suficiente, a no ser que den lugar a la acción transformadora, responsable y sostenible. Se ha determinado la complejidad del Pensamiento crítico, en cuanto se ha evidenciado las diversas dimensiones que se involucran en él. Los 
métodos tradicionales de enseñanza - aprendizaje se encuentras desfasados en el tiempo. El nuevo contexto social, político, cultural, etc., exige que la educación se limite a fomentar el memorismo y busca impulsar un proceso que se adapta a las distintas asignaturas curriculares, sea ciencias o sociales, o se trate de despertar el espíritu creativo de los estudiantes, éstos sin estudiantes con pensamiento crítico serán meros contenidos.

\section{CONCLUSIONES}

En conformidad con los hallazgos de los artículos selecciónalos y, debidamente, analizados, se puede apreciar que la preocupación por el desarrollado del pensamiento crítico en la educación básica, es compartida por muchos países del mundo, ya que se ha establecido su vital importancia como parte esencial en el proceso de enseñanza aprendizaje. De su comprensión y aprehensión dependerá un sistema educativo de calidad que contribuirá de manera significativa al logro de todas las capacidades, misión y visión de las instituciones educativas.

De las investigaciones consultadas, se ha determinado que los profesionales de la educación han centrado su interés en proporcionar diversas propuestas de cómo se he de direccionar el proceso de enseñanza - aprendizaje a un contexto donde los alumnos sean capaces de pensar críticamente desde muy temprana edad, con la finalidad de que esta adquisición de habilidades les facilite hacer frente a los diversos contextos en donde se desenvuelve, convirtiéndose en ciudadanos productivos y empáticos.

Las investigaciones evidencian que los estudiantes carecen de las habilidades necesarias para la comprensión de textos; es decir, los estudiantes presentan muchas dificultades al analizar, deducir, concluir y tomar decisiones. Siendo estos resultados opuestos a los establecidos como objetivos de aprendizaje en las aulas del nivel primaria, se espera que los estudiantes, a través de la educación impartida sean capaces de observar, describir, interpretar y tomar decisiones ante una problemática; es decir que, sus habilidades le sean útiles en las distintas áreas de aprendizaje.

El desarrollo del pensamiento crítico facilitará a los estudiantes a tomar sus decisiones en base al previo análisis del contexto, en la evaluación de la información y de las distintas alternativas de solución que podrían distinguir, dejando de lado sus preferencias personales y enfocarse a la fundamentación de sus elecciones por medio del criterio lógico. De ahí la importancia de promover este desarrollado en el nivel de educación primaria, identificando las fortalezas y debilidades del desempeño de estas habilidades en los estudiantes. 
De lo mencionado, surge la propuesta de diversos programas que direccionen al estudiante de nivel primaria a un fortalecimiento de sus habilidades que lo conduzcan a un aprendizaje autónomo y que impulsen habilidades específicas vinculadas al pensamiento crítico, tales como la reflexión sistemática, el análisis crítico, el desarrollo de la creatividad, el compromiso con la tarea del pensar y la sensibilidad con el contexto cultural y social.

\section{REFERENCIAS BIBLIOGRÁFICAS}

Aguirre, F.; Bouchon, M., Juárez, D., y Chacara, M. (2020). Taller fotográfico cultura y turismo en el pensamiento crítico de estudiantes de la isla Amantani. Revista Ibérica De Sistemas e Tecnologias De Informação, 539-555. https://search.proquest.com/scholarly-journals/taller-fotográfico-cultura-yturismo-en-

Botero, A., Alarcón, D.; Palomino, D. y Jiménez, A. (2017). Pensamiento crítico, metacognición y aspectos motivacionales: una educación de calidad. Revista Poiésis, (33), pp. 85-103. DOI: https://doi.org/10.21501/16920945.2499.

Castillo, R (2020). El Pensamiento Crítico como competencia básica. Una propuesta de nuevos estándares pedagógicos. Ixtli. Revista Latinoamericana de Filosofía de la Educación. 7(14).127-148.

Chrobak, R. (2017). El aprendizaje significativo para fomentar el pensamiento crítico. Revista Archivos de Ciencias de la Educación, 11(12), e031

Collazo, L. (2017). Avanzar en la educación para la sostenibilidad. Combinación de metodologías para trabajar el pensamiento crítico y autónomo, la reflexión y la capacidad de transformación del sistema. Revista Iberoamericana de Educación [(2017), vol. 73, pp. 131-154] - OEI/CAEU

Correa, J.; Ossa, C. y Sanhueza, P. (2019). Reasoning bias, metacognition and motivation for critical thinking in freshmen middle of a establishment of Chillán. Revista de Estudios y Experiencias en Educación, vol. 18, núm. 37.

Del Barrio, L. (2017). El Pensamiento Crítico Como Estrategia Didáctica Musical En La Educación Emancipatoria Del Alumno En Educación Primaria. European Scientific Journal; Dec2017, Vol. 13 Issue 35, p37-52, 16p

Deroncele, A.; Nagamine, M. y Medina, D. (2020) Bases epistemológicas y metodológicas para el abordaje del pensamiento críticos en la educación peruana. 
Revista de Humanidades y Ciencias Sociales. Volumen 7. Número Especial ISSN 0719-4706.

Godoy, F. (2017). Enseñanza de la historia escolar. Un aporte al desarrollo del pensamiento crítico. Revista Clio \& Asociados $\mathrm{N}^{\circ} 25$ pp. 34-46

Gómez, D. y De la Herrán, A. (2018). Development of critical thinking in secondary school students: design, application and evaluation of an educational program. Revista de currículum y formación del profesorado Vol. 22. N4 ISSN 1138-414X.

Grez, F. (2018). Veo, pienso y me pregunto. El uso de rutinas de pensamiento para promover el pensamiento crítico en las clases de historia a nivel escolar. Revista Praxis Pedagógica 18(22), 65-84. doi: 10.26620/uniminuto.praxis.18.22.2018.6584

Guerrero, H.; Polo, S.; Martínez, J: y Ariza, P. (2018) Trabajo colaborativo como estrategia didáctica para el desarrollo del pensamiento crítico. Revista Opción, año $34 N^{\circ} 86$ ISSN 1012-158.

Habowski,A. y Conte, E.(2020). Interacciones crítico dialécticas con las tecnologías en la educación. Revista Ibero-Americana de Estudos em Educação, Araraquara, v. 15, n. 1, p. 266-288, jan./mar. 2020. E-ISSN: 1982-5587.DOI: https://doi.org/10.21723/riaee.v14i4.11993

Huanca, J. y Canaza, F. (2019). Puno: Educación rural y pensamiento crítico. Hacia una educación inclusiva. Revista Helios, 3 (1), 97-108

Lecturer, M.; Sutansi, S.; Ayu, M. y Kistin, R. (2019) Strengthening critical thinking of students for education teachers primary school through the development of basic math textbooks based on skills to life and problem solving.. Revista Dilemas Contemporáneos: Educación, Política y Valores. Año VII, Edición

DOI: https://doi.org/10.46377/dilemas.v30i1.1200.

Lévano, S. (2020). Critical thinking and acquisition of strategic competence in translation students. Revista Educación XXIX ISSN 1019-9403.

Loaiza, Y. \& Delia, L. (2018) The development of critical thinking in natural sciences with elementary school students in an Educational Institution in Pereira Risaralda.Revista Diálogos sobre educación. Temas actuales en investigación educativa, 9 (16), 00009. 
López, R. y Villa, G. (2017). The design classroom as an exploration scenario between visual literacy and critical thinking. Revista KEPES Año 14 No. 15 págs. 173-194 ISSN: 1794-7111 DOI: 10.17151/kepes.2017.14.15.7

Madrid H., J. (2018). Desarrollo del pensamiento crítico desde el área de Ciencias Sociales en la educación básica secundaria. Revista Praxis Pedagógica 18(22), 4964. doi: 10.26620/uniminuto.praxis.18.22.2018.49-64.

Martínez, A., Cabrera, H., Borjas, M., Torres, E., y Judex, J. (2018). Evaluando la disposición y la motivación del pensamiento crítico con la mediación de las TIC. Revista Praxis, 14(2), 187-203. http://dx.doi.org/10.21676/23897856.2762

Martínez, D. (2017). Evaluar el pensamiento crítico en educación para la ciudadanía. Propuesta para contextos masificados. Revista DIDACTICAE Universitat de Barcelona ISSN 2462-2737 DOI: 10.1344/did.2018.3.131-144

Meneses, J.; Osorio, K. y Rubio, A. (2018). La comprensión de textos argumentativos como estrategia para el aprendizaje significativo y el desarrollo del pensamiento crítico. Revista Actualidades Pedagógicas, (72), 29-47. doi: https://doi.org/10.19052/ap.4336

Minte, A.; e Ibagón, N. (2017) Pensamiento crítico: ¿competencia olvidada en la enseñanza de la historia? Revista Entramado. vol. 13, no. 2, p. 186-198 http://dx.doi.org/10.18041/entramado.2017v13n2.26228

Moreno, W. y Velázquez, M. (2017) Estrategia Didáctica para Desarrollar el Pensamiento Crítico. REICE. Revista Iberoamericana sobre Calidad, Eficacia y Cambio en Educación, 15(2), 53-73. https://doi.org/10.15366/reice2017.15.2.003

Núñez, A., Gallardo, M., Aliaga, A. y Díaz, J. (2020). Didactic strategies in the development of thought. Revista Eleuthera, 22 (2), 31-50. DOI: 10.17151/eleu.2020.22.2.3.

Ortega, V. y Gil, C. (2019). La naturaleza de la ciencia y la tecnología. Una experiencia para desarrollar el pensamiento crítico. Revista Científica,35(2), pp. 167-182.

Prieto, F. (2018) El pensamiento crítico y autoconocimiento. Revista de Filosofía Volumen 74 (2018) 173-191

Ríos, O. (2017). Development of critical thinking skills in the social sciences through the implementation of graphic organizers in rural secondary education. Revista Assensus, 2(2), 83-98. https://doi.org/10.21897/assensus.1323 
Rodríguez, A. (2018) Elementos ontológicos del pensamiento crítico. Revista Interuniversitaria, 30(1), 53-20.

Rojas J., Pérez H. y Álvarez A. (2017). El pensamiento crítico en la educación. Revista Publicando, 3(9).2016, 110-118. ISSN 1390-9304

Ros, I. Ortiz, I. y Zelaieta, E (2020) Student engagement and critical thinking - creative Revista Contextos de Educación $\mathrm{N}^{\mathrm{o}} 25$. Pp 67-76

Sálica, M. (2018) Characterization of critical thinking skills for the development of didactic content knowledge in Natural Sciences teachers. Revista Enseñanza \& Teaching; $\quad$ Salamanca Vol. 36, N. ${ }^{\circ} 1, \quad$ pp $\quad 199-221$ DOI:10.14201/et2018361199221

Sánchez, M. Martínez, C. Águila, E: y Cáceres, J. (2017) Habilidades y estrategias para el desarrollo del pensamiento crítico y creativo. Revista de educación Vol. 1 ISSN 1853-1318.

Silva, C. (2019). El desarrollo del pensamiento crítico en la propuesta curricular de la educación del arte en Chile. Revista de Estudios Pedagógicos XLV, $\mathrm{N}^{\circ}$ 3: 79-92, 2019 DOI: $10.4067 /$ S0718-07052019000300079

Standish, P. y Thoilliez, B. (2018) El pensamiento crítico en crisis. una reconsideración pedagógica en tres movimientos. Revista Interuniversitaria; Salamanca Tomo 30, N. ${ }^{\circ}$, pp. 7

Steffens, E., Ojeda, D., Martínez, J. y Hernández, H. (2018). Presencia del pensamiento crítico en estudiantes de educación superior de la Costa Caribe Colombiana. Revista Espacios ISSN 07981015.

Tamayo, O., Loaiza Y. y Ruiz F.(2020). Hacia la construcción de un modelo de pensamiento crítico dominio específico. Poliésis, Revista do programa de pósgraducao-mestrado

Torres, D.; Fonseca, W. y Pineda, B. (2017). Las vivencias como estrategia de fortalecimiento del pensamiento crítico en educación rural. Revista Praxis \& Saber; Tunja Tomo 8, N..$^{\circ} 17$ 201224. DOI:10.19053/22160159.v8.n17.2018.7207.

Zelaieta, E., Camino, I., Zulaika, L. y Echeazarra, I.(2018). Juego de rol para el desarrollo del pensamiento crítico en la formación inicial del profesorado y aspectos 
Pensamiento crítico en estudiantes de...

motivacionales: uma educación de calidad. Revista Complutense de Educación ISSNe: 1988-2793 http://dx.doi.org/10.5209/RCED.58884 